\title{
Adaptive Inverse Optimal Neuromuscular Electrical Stimulation
}

\author{
Qiang Wang, Nitin Sharma, Marcus Johnson, Member, IEEE, Chris M. Gregory, and \\ Warren E. Dixon, Senior Member, IEEE
}

\begin{abstract}
Neuromuscular electrical stimulation (NMES) is a prescribed treatment for various neuromuscular disorders, where an electrical stimulus is provided to elicit a muscle contraction. Barriers to the development of NMES controllers exist because the muscle response to an electrical stimulation is nonlinear and the muscle model is uncertain. Efforts in this paper focus on the development of an adaptive inverse optimal NMES controller. The controller yields desired limb trajectory tracking while simultaneously minimizing a cost functional that is positive in the error states and stimulation input. The development of this framework allows tradeoffs to be made between tracking performance and control effort by putting different penalties on error states and control input, depending on the clinical goal or functional task. The controller is examined through a Lyapunov-based analysis. Experiments on able-bodied individuals are provided to demonstrate the performance of the developed controller.
\end{abstract}

Index Terms-Functional electrical stimulation (FES), inverse optimal control, Lyapunov stability, neural network (NN), neuromuscular electrical stimulation, nonlinear system control.

\section{INTRODUCTION}

$\mathbf{N}$ EUROMUSCULAR electrical stimulation (NMES) (also known as functional electrical stimulation (FES), particularly when eliciting functional tasks) has the potential to facilitate improved functionality in persons with certain neurological disorders [1]-[3] (e.g., stroke, spinal injuries, etc.). As a rehabilitation tool, traditional NMES/FES methods are open-loop approaches used to strengthen muscle [4]. With advances in and application of feedback control, new rehabilitative capabilities

Manuscript received February 10, 2012; revised July 11, 2012; accepted October 20, 2012. Date of publication January 9, 2013; date of current version November 18, 2013. This work was supported in part by the National Science Foundation under Grant 0547448, Grant 0901491, and Grant 1161260; and in part by The Air Force Research Laboratory Munitions Directorate. This paper was recommended by Associate Editor H. Zhang.

Q. Wang is with the Department of Electrical and Computer Engineering, University of Florida, Gainesville, FL 32611 USA (e-mail: qiangw@ufl.edu).

N. Sharma is with the Department of Mechanical Engineering and Materials Science, University of Pittsburgh, PA 15261 USA (e-mail: nis62@ pitt.edu).

M. Johnson was with the Department of Mechanical and Aerospace Engineering, University of Florida, Gainesville, FL 32611 USA. He is now with the Ames Research Center, National Aeronautics and Space Administration, Moffett Field, CA 94035 USA (e-mail: marc1518@ufl.edu).

C. M. Gregory is with Ralph H. Johnson Veterans Affairs Medical Center, Charleston, SC 29401 USA and also with the Department of Health Sciences and Research, The Medical University of South Carolina, Charleston, SC 29425 USA (e-mail: gregoryc@musc.edu).

W. E. Dixon is with the Department of Electrical and Computer Engineering and the Department of Mechanical and Aerospace Engineering, University of Florida, Gainesville, FL 32611 USA (e-mail: wdixon@ufl.edu).

Color versions of one or more of the figures in this paper are available online at http://ieeexplore.iee.org.

Digital Object Identifier 10.1109/TSMCB.2012.2228259 are possible, such as accurate and precise limb motions for gait retraining [5].

The development of closed-loop NMES controllers is challenging because the muscle response is inherently nonlinear and the muscle activation and limb dynamics contain parametric and unstructured uncertainty. Several proportional integral derivative (PID)-based NMES controllers have been developed (cf. [6]-[9] and the references within), but these methods typically either lack a stability analysis or are based on a linear muscle model. Neural network (NN)-based NMES controllers (cf. [10]-[20]) have been also developed based on the idea that the universal approximation property of NNs can be used to approximate the nonlinear (unstructured) dynamics. Robust NMES methods have been also recently developed in [21] and [22], which achieve guaranteed asymptotic limb tracking.

Closed-loop control of muscle has been proven to yield accurate limb positioning, but continuous external stimulation of muscle can lead to rapid fatigue (particularly if the controller requires high gains to include robustness to disturbances/ uncertainty in the dynamics). Rehabilitative procedures seek to maximize the number of repetitive steps; thus, muscle fatigue is a critical concern. While various stimulation strategies have been investigated (cf. [23]-[26]) such as choosing different stimulation patterns and parameters, improving fatigue resistance through muscle retraining, sequential stimulation, and size order recruitment, reducing the onset of fatigue remains a largely open research topic.

As closed-loop NMES continues to become part of the suite of available rehabilitative tools, one question to consider is the balance between stimulation and the resulting performance. Is it better to use less control authority for potentially less fatigue and more tracking error, or is it better to maintain low tracking errors with greater control authority at the expense of potentially faster fatigue? As researchers from various fields investigate the answer to such questions, there is a need for a control framework to examine such tradeoffs. The contribution in this paper is the formulation of an inverse optimal control framework where a stabilizing proportional- derivative controller is shown to minimize a meaningful cost functional of the limb tracking errors and control input for the uncertain nonlinear muscle model.

Nonlinearities in the system dynamics pose challenges in developing controllers that can guarantee both stability and minimization of cost function. Inverse optimal control [27], [28] is used to avoid the complexity of solving the steadystate Hamilton-Jacobi-Bellman equations. Rather than minimizing a given cost functional, inverse optimal control aims to 
parameterize a family of stabilizing controllers that minimize a meaningful derived cost functional. The derived cost functional is meaningful in the sense that it puts a positive penalty on the states and control input.

As in our preliminarily results in [29], an NMES controller with associated stability analysis is developed, which yields limb trajectory tracking while minimizing a cost functional of the error states and control effort (and hence, an attempt to strike a balance between performance and control stimulation). In particular, an adaptive inverse optimal NMES controller is developed for lower limb trajectory tracking in the presence of parametric uncertainty and external disturbances in the muscle activation and limb dynamics model. A Lyapunovbased stability analysis is used to prove that the developed NN-based controller yields semiglobal uniformly ultimately bounded (SUUB) tracking while simultaneously minimizing a cost functional. Experiments on able-bodied volunteers validate the performance of the proposed controller for a limb tracking task, a step response, and a sit-to-stand-like task to illustrate changing loads. Experiments also illustrate the ability to alter the control performance through changing the weights (i.e., control parameters in the cost functional).

\section{Muscle Activation And Limb Model}

The dynamics of a free swinging shank when the subject is seated can be segregated into body segmental dynamics and muscle activation and contraction dynamics. The complete dynamic model is given by [30]

$$
M_{I}+M_{e}+M_{g}+M_{v}+\tau_{d}=\tau .
$$

In (1), $M_{I}(\ddot{q}) \in \mathbb{R}$ denotes the inertial effects of the shank-foot complex about the knee joint; $M_{e}(q) \in \mathbb{R}$ denotes the nonlinear elastic effects due to joint stiffness; $M_{g}(q) \in \mathbb{R}$ denotes the gravitational component; $M_{v}(\dot{q}) \in \mathbb{R}$ denotes the nonlinear viscous effects due to damping in the musculotendon complex [31]; $\tau_{d}(t) \in \mathbb{R}$ is considered as an unknown bounded disturbance, which represents an unmodeled reflex activation of the muscle (e.g., muscle clonus and spasticity) and other unknown unmodeled phenomena (e.g., fatigue); and $\tau(t) \in \mathbb{R}$ denotes the torque produced at the knee joint, where $q(t), \dot{q}(t), \ddot{q}(t) \in \mathbb{R}$ denote the generalized angular position, the velocity, and the acceleration of the lower limb about the knee joint, respectively. The inertial component $M_{I}(q) \in \mathbb{R}$ is defined as

$$
M_{I}(\ddot{q}(t))=J \ddot{q}(t) .
$$

The elastic effects are modeled on the empirical findings by Ferrarin and Pedotti [31] as

$$
M_{e}=-k_{1}\left(e^{-k_{2} q}\right)\left(q-k_{3}\right)
$$

where $k_{1}, k_{2}, k_{3} \in \mathbb{R}$ are unknown positive coefficients. As shown in [30], the viscous moment $M_{v}(\dot{q})$ can be modeled as

$$
M_{v}=B_{1} \tanh \left(-B_{2} \dot{q}\right)-B_{3} \dot{q}
$$

where $B_{1}, B_{2}, B_{3} \in \mathbb{R}$ are unknown positive constants. The torque produced at the knee joint can be modeled as

$$
\tau(t)=\zeta V(t)
$$

where $V(t) \in \mathbb{R}$ is the electrical stimulus applied to the quadriceps muscle group, and $\zeta(q, \dot{q}) \in \mathbb{R}$ is a mapping function between the generated knee torque and the applied electrical stimulus on the quadriceps muscle group. For complete details of the dynamics in (1), see [22].

Assumption 1: Based on the results in [32], the nonlinear function $\zeta(q, \dot{q})$ is assumed to be a continuously differentiable, positive, and bounded function.

Assumption 2: The disturbance term $\tau_{d}(t)$ and its first time derivative are assumed to be bounded. This assumption is reasonable for typical disturbances such as muscle spasticity, fatigue, and load changes during functional tasks.

To facilitate the subsequent analysis, the expression in (1) is rewritten as

$$
J_{\zeta} \ddot{q}(t)+M_{\zeta}+\tau_{d \zeta}=V(t)
$$

where $J_{\zeta}(q, \dot{q}), M_{\zeta}(q, \dot{q}), \tau_{d \zeta}(q, \dot{q}) \in \mathbb{R}$ are defined as

$$
J_{\zeta}=\zeta^{-1} J, M_{\zeta}=\zeta^{-1}\left(M_{e}+M_{g}+M_{v}\right), \tau_{d \zeta}=\zeta^{-1} \tau_{d} .
$$

Based on Assumptions 1 and 2, the following inequalities can be developed:

$$
\xi_{0} \leq\left|J_{\zeta}\right| \leq \xi_{1}, \quad\left|\tau_{d \zeta}\right| \leq \xi_{2}
$$

where $\xi_{0}, \xi_{1}, \xi_{2} \in \mathbb{R}$ are known positive constants.

\section{Control Development}

A rehabilitative goal of NMES is to elicit a desired muscle response that can lead to restored independent function. For rehabilitative outcomes, repetitive training is essential; however, electrically stimulated muscle can often fatigue quickly due to overstimulation and various other factors such as synchronous excitation and nonphysiological motor unit recruitment order. As an inroad to address these concerns, the control objective is to stimulate the quadriceps muscle group to enable the shank to track a desired time-varying trajectory, which is denoted by $q_{d}(t) \in \mathbb{R}$, despite uncertainties in the dynamic model, while also minimizing a given performance index that includes a penalty on the tracking error and the control effort.

To quantify the tracking objective, a lower limb angular position tracking error and an auxiliary tracking error denoted by $e(t), r(t) \in \mathbb{R}$, respectively, are defined as

$$
e=q_{d}-q, \quad r=\dot{e}+\alpha e
$$

where $\alpha \in \mathbb{R}$ is a positive constant gain.

After taking the time derivative of $r(t)$, multiplying it by $J_{\zeta}(q, \dot{q})$, and utilizing (6) and (9), the following open-loop error system can be obtained:

$$
J_{\zeta} \dot{r}=\tau_{d \zeta}+f_{1}+f_{2}-V
$$

where $f_{1}(t), f_{2}(t) \in \mathbb{R}$ are defined as

$$
f_{1}=J_{\zeta} \alpha \dot{e}, \quad f_{2}=J_{\zeta} \ddot{q}_{d}+M_{\zeta} .
$$


Based on (10) and the subsequent stability analysis (given in Theorem 1), the voltage control input $V(t)$ is designed as

$$
V=u_{2}-u_{1}=\hat{f}_{2}-u_{1}
$$

where $u_{1}(t) \in \mathbb{R}$ is a subsequently designed control input, and $u_{2}(t)=\hat{f}_{2}(t) \in \mathbb{R}$ is an NN estimate of $f_{2}(t)$ (see Fig. 1). A three-layer $\mathrm{NN}$ can be used to represent $f_{2}$ as

$$
f_{2}(y)=W^{T} \sigma\left(U^{T} y\right)+\epsilon(y)
$$

where $U \in \mathbb{R}^{\left(N_{1}+1\right) \times N_{2}}$ and $W \in \mathbb{R}^{\left(N_{2}+1\right) \times 1}$ are bounded constant ideal weight matrices, $\sigma(\cdot): \mathbb{R}^{N_{1}+1} \rightarrow \mathbb{R}^{N_{2}+1}$ is an NN activation function, $y(t) \in \mathbb{R}^{N_{1}+1}$ is an input vector defined as

$$
y(t)=\left[1 q(t) \dot{q}(t) \ddot{q}_{d}(t)\right]^{T}
$$

and $\epsilon(y): \mathbb{R}^{N_{1}+1} \rightarrow \mathbb{R}$ is a functional reconstruction error that can be upper bounded as

$$
|\epsilon(y)| \leq \delta
$$

where $\delta \in \mathbb{R}$ is a known positive constant. The estimate $\hat{f}_{2}(t)$ is designed as

$$
\hat{f}_{2}=\hat{W}^{T} \sigma\left(\hat{U}^{T} y\right)
$$

where $\hat{U}(t) \in \mathbb{R}^{\left(N_{1}+1\right) \times N_{2}}$ and $\hat{W}(t) \in \mathbb{R}^{\left(N_{2}+1\right) \times 1}$ are weight estimate matrices. The ideal weight matrix estimates $\hat{U}(t)$ and $\hat{W}(t)$ are updated online using the following projection algorithm:

$$
\dot{\hat{W}}=\operatorname{proj}\left(\Gamma_{w} \hat{\sigma} r^{T}\right), \quad \dot{\hat{U}}=\operatorname{proj}\left(\Gamma_{u} y\left(\hat{\sigma}^{\prime T} \hat{W} r\right)^{T}\right)
$$

where $\Gamma_{w} \in \mathbb{R}^{\left(N_{2}+1\right) \times\left(N_{2}+1\right)}$ and $\Gamma_{u} \in \mathbb{R}^{\left(N_{1}+1\right) \times\left(N_{1}+1\right)}$ are constant, positive definite, and symmetric gain matrices; $\hat{\sigma}=$ $\sigma\left(\hat{U}^{T} y\right)$; and $\hat{\sigma}^{\prime}=\sigma^{\prime}\left(\hat{U}^{T} y\right)=d \sigma\left(U^{T} y\right) / d\left(U^{T} y\right)_{\mid U^{T} y=\hat{U}^{T} y}$.

The weight mismatch errors $\tilde{U}(t) \in \mathbb{R}^{\left(N_{1}+1\right) \times N_{2}}$ and $\tilde{W}(t) \in \mathbb{R}^{\left(N_{2}+1\right) \times 1}$ are denoted by

$$
\tilde{W}=W-\hat{W}, \quad \tilde{U}=U-\hat{U}
$$

and the hidden-layer output mismatch $\tilde{\sigma}(y) \in \mathbb{R}^{N_{2}+1}$ for a given $y(t)$ is defined as

$$
\tilde{\sigma}=\sigma-\hat{\sigma}=\sigma\left(U^{T} y\right)-\sigma\left(\hat{U}^{T} y\right) .
$$

By using a Taylor series approximation, the hidden-layer output mismatch $\tilde{\sigma}(y)$ can be expressed as

$$
\tilde{\sigma}=\hat{\sigma}^{\prime} \tilde{U}^{T} y+O\left(\tilde{U}^{T} y\right)^{2}
$$

where $O\left(\tilde{U}^{T} y\right)^{2}$ denotes the higher order terms.

Substituting (12) into (10) and performing some algebraic manipulation yields

$$
J_{\zeta} \dot{r}=N+\tilde{W}^{T} \hat{\sigma}+\hat{W}^{T} \hat{\sigma}^{\prime} \tilde{U}^{T} y+u_{1}
$$

where the auxiliary term $N(\tilde{W}, \tilde{U}, y) \in \mathbb{R}$ is defined as

$$
N=f_{1}+\tilde{W}^{T} \hat{\sigma}^{\prime} \tilde{U}^{T} y+W^{T} O\left(\tilde{U}^{T} y\right)^{2}+\epsilon(y)+\tau_{d \zeta} .
$$

Based on (8), (15), and (17), $N(\tilde{W}, \tilde{U}, y)$ can be upper bounded as [33]

$$
\|N\| \leq c_{1}+c_{2}\|z\|
$$

where $c_{1}, c_{2} \in \mathbb{R}$ are known positive constants, and $z(t) \in \mathbb{R}^{2}$ is defined as

$$
z(t)=\left[\begin{array}{ll}
e & r
\end{array}\right]^{T} .
$$

Based on (10) and the subsequent stability analysis, the stabilizing PD controller $u_{1}$ in (21) is designed as

$$
u_{1}=-R^{-1} r=-\left(k_{s_{1}}+k_{s_{2}}+k_{s_{3}}\right) r
$$

where $R^{-1}, k_{s_{1}}, k_{s_{2}}, k_{s_{3}} \in \mathbb{R}$ denote positive adjustable gains.

From Assumption 1, (10)-(12), and (25), it can be shown that

$$
\frac{1}{2}\left|\dot{J}_{\zeta}\right| \leq \rho(\|z\|)
$$

where $\rho(\|z\|) \in \mathbb{R}$ is a positive global invertible function.

\section{STABILITy ANALYSis}

Theorem 1: The controller given in (12), (16), and (25) ensures that all closed-loop signals are bounded, and the position tracking error is SUUB in the sense that

$$
|e(t)| \leq \epsilon_{0} \exp \left(-\epsilon_{1} t\right)+\epsilon_{2}
$$

where $\epsilon_{0}, \epsilon_{1}, \epsilon_{2} \in \mathbb{R}$ denote positive constants in $\mathcal{D} \triangleq\{z \subset$ $\left.\left.\mathbb{R}^{2} \mid\|z\| \leq \rho^{-1}\left(\sqrt{k_{s_{3}}}\right)\right)\right\}$, provided the control gains $\alpha$ and $k_{s 2}$ introduced in (9) and (25) are selected based on the following sufficient conditions:

$$
\min \left(k_{s_{2}}-\frac{1}{2}, \alpha-\frac{1}{2}\right)>c_{2}, \quad k_{s_{2}}, \alpha>\frac{1}{2} .
$$

Proof: Consider a positive definite, continuously differentiable, and radially unbounded function $V_{L}(e, r, \tilde{W}, \tilde{U}) \in \mathbb{R}$ defined as

$V_{L}=\frac{1}{2} e^{2}+\frac{1}{2} J_{\zeta} r^{2}+\frac{1}{2} \operatorname{tr}\left(\tilde{W}^{T} \Gamma_{w}^{-1} \tilde{W}\right)+\frac{1}{2} \operatorname{tr}\left(\tilde{U}^{T} \Gamma_{u}^{-1} \tilde{U}\right)$.

By using (8) and typical NN properties [34], $V_{L}(t)$ can be upper and lower bounded as

$$
\gamma_{1}\|z\|^{2} \leq V_{L} \leq \gamma_{2}\|z\|^{2}+\gamma_{3}
$$

where $\gamma_{1}, \gamma_{2}, \gamma_{3} \in \mathbb{R}$ are known positive constants. Taking the time derivative of (29), utilizing (17) and (21), and canceling common terms yield

$$
\dot{V}_{L}=e \dot{e}+\frac{1}{2} \dot{J}_{\zeta} r^{2}+r N+r u_{1} .
$$

Using (9) and Young's inequality, the expression in (31) can be bounded as

$$
\dot{V}_{L} \leq-\left(\alpha-\frac{1}{2}\right) e^{2}+r^{2}\left(\frac{1}{2} \dot{J}_{\zeta}+\frac{1}{2}\right)+r N+r u_{1} .
$$


By utilizing (23), (25), and (26), the expression in (32) can be upper bounded as

$$
\begin{aligned}
\dot{V}_{L} \leq & -\left(\alpha-\frac{1}{2}\right) e^{2}-\left(k_{s_{2}}-\frac{1}{2}\right) r^{2} \\
& -\left(k_{s_{1}} r^{2}-c_{1}|r|\right)+c_{2}\|z\|^{2}-\left(k_{s_{3}}-\rho(\|z\|) r^{2} .\right.
\end{aligned}
$$

Applying nonlinear damping and neglecting negative terms, the expression in (33) can be upper bounded as

$$
\dot{V}_{L} \leq-\gamma_{4}\|z\|^{2}+\frac{c_{1}^{2}}{4 k_{s_{1}}}, \quad \forall\|z\| \in \mathcal{D}
$$

and $\gamma_{4}=\min \left(k_{s_{2}}-(1 / 2), \alpha-(1 / 2)\right)-c_{2}>0$, provided the sufficient gain conditions in (28) are satisfied. The inequality in (30) can be used to rewrite (34) as

$$
\dot{V}_{L} \leq-\frac{\gamma_{4}}{\gamma_{2}} V_{L}+\varepsilon, \quad \forall\|z\| \in \mathcal{D}
$$

where $\varepsilon \in \mathbb{R}$ is a positive constant. The linear differential inequality in (35) can be solved as

$$
V_{L}(t) \leq V_{L}(0) e^{-\frac{\gamma_{4}}{\gamma_{2}} t}+\varepsilon \frac{\gamma_{2}}{\gamma_{4}}\left[1-e^{-\frac{\gamma_{4}}{\gamma_{2}} t}\right], \quad \forall\|z\| \in \mathcal{D} .
$$

Provided the sufficient conditions given in (28) are satisfied, the expressions in (29) and (36) can be used to prove that the control input and all the closed-loop signals are bounded in $\mathcal{D}$. A larger value of $k_{s_{3}}$ and $k_{s_{1}}$ will expand the size of the domain $\mathcal{D}$ to include any initial conditions (i.e., a semiglobal type of stability result) and reduce the residual error. From (29) and (36), the result in (27) can be obtained.

\section{Cost Functional Minimization}

An inverse optimal controller [27], [35], [36] is optimal with respect to an a posteriori cost functional that is derived from a Lyapunov-based analysis (in comparison with minimizing an a priori given cost functional in direct optimal control). Due to the use of an NN to compensate for the unstructured uncertainty in the muscle model, a residual disturbance is present in the system (i.e., the UUB stability result). Given this residual disturbance, the succeeding analysis is formulated in the spirit of a two-player zero-sum differential game, where the objective is to minimize the cost functional with respect to the control input in the presence of the maximum "worst case" disturbance. The feedforward NN element estimates the uncertain dynamics, whereas the feedback element is penalized by the cost functional.

Theorem 2: The feedback law given by

$$
u_{o}^{*}=-\beta R^{-1} r
$$

with the scalar gain constant selected as $\beta>2$ and the update law given in (17), minimizes the cost functional

$$
J=\lim _{t \rightarrow \infty}\left\{2 \beta V_{L}(t)+\int_{0}^{t}\left[l+u_{1}^{2} R\right]-2 \beta \frac{c_{1}^{2}}{4 k_{s_{1}}} d \sigma\right\}
$$

where $l(z, t) \in \mathbb{R}$ is a positive function of the tracking error

$$
l=-2 \beta\left(e \dot{e}+\frac{1}{2} \dot{J}_{\zeta} r^{2}+r N-\frac{c_{1}^{2}}{4 k_{s_{1}}}\right)+\beta^{2} r^{2} R^{-1}
$$

provided the sufficient conditions in (28) are satisfied.

Proof: The cost functional in (38) is said to be meaningful if the bracketed terms are positive (i.e., positive state and control functions). To examine the sign of $l(z, t)$, the expressions in (25), (31), and (34) and the condition in (28) can be used to determine that

$$
e \dot{e}+\frac{1}{2} \dot{J}_{\zeta} r^{2}+r N-\frac{c_{1}^{2}}{4 k_{s_{1}}}-r^{2} R^{-1} \leq 0 .
$$

After multiplying both sides by $-2 \beta$ and adding $\beta(\beta-$ 2) $r^{2} R^{-1}$ to both sides of (40), the expression in (40) can be rewritten as

$$
l \geq \beta(\beta-2) r^{2} R^{-1}=Q r^{2}
$$

where $Q \in \mathbb{R}$ is a positive constant. The inequality in (41) indicates that $l(z, t)$ is positive since $R$ is positive and $\beta>2$. Therefore, $J(t)$ is a meaningful cost functional that penalizes the error function in $z(t)$ and the feedback control $u_{1}(t)$. The cost functional in (38) and the result in (41) indicate that larger values of $Q$ place a greater penalty on the tracking error, whereas larger values of $R$ place a greater penalty on the feedback control. The effects of selecting different values for $Q$ and $R$ are illustrated in Section VI.

To show that $u_{o}^{*}$ minimizes $J(t)$, the auxiliary signal $v(t) \in$ $\mathbb{R}$ is defined as

$$
v=u_{1}+\beta R^{-1} r .
$$

Substituting (39) and (42) into (38) and performing some algebraic manipulation yield

$$
J=\lim _{t \rightarrow \infty}\left\{2 \beta V_{L}(t)+\int_{0}^{t} v^{2} R d \sigma-2 \beta \int_{0}^{t} \dot{V}_{L} d \sigma\right\} .
$$

After integrating (43), the cost functional $J(t)$ can be expressed as

$$
J=2 \beta V_{L}(0)+\lim _{t \rightarrow \infty}\left\{\int_{0}^{t} v^{2} R d \sigma\right\} .
$$

By substituting (37) into (31), it can be shown that $u_{o}^{*}$ stabilizes the system. Since $J(t)$ is minimized if $v(t)=0$, the control law $u_{1}=u_{o}^{*}$ is optimal with respect to the meaningful cost functional in (38).

\section{EXPERIMENT RESULTS}

The proposed inverse optimal controller was implemented on healthy normal volunteers to evaluate the performance of the controller. The focus of this paper is to develop and analyze an inverse optimal controller as a means to provide a method for understanding the tradeoff of the control parameters $Q$ and $R$ 


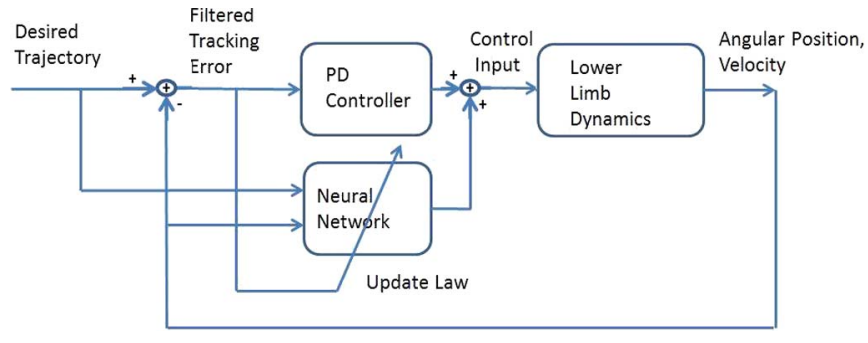

Fig. 1. Structure of the controller.

that are included in a cost functional composed of terms such as the limb tracking error and stimulation input terms. This section describes the performance of the developed strategy when implemented on a group of healthy normal volunteers. The performance of the developed method may vary when implemented in populations of individuals affected with different neurological disorders: Clinical trials on specific affected populations of interest are motivated as future work to further the clinical implications of the succeeding outcomes. The results obtained from healthy normal subjects here may provide some insights into further clinical trials. For example, in [37] (and in results such as [38]-[40], which directly or indirectly cite the work in [37]), a relaxed limb is shown to behave like a recently paralyzed limb. However, there are differences in the muscle response, which are associated with different conditions. For example, a limb that has been paralyzed for some time will exhibit muscle atrophy with disuse, will fatigue more rapidly, and may exhibit clonus and muscle spasticity [39]. The adaptive structure of the controller compensates for effects such as lower force outputs (e.g., due to fatigue or muscle atrophy). The controller has been also developed and analyzed while including added unmodeled disturbances which could be used to capture the effects of clonus and spasticity. However, the aforementioned effects for paralyzed muscle are not present in the healthy normal volunteer subjects. Ablebodied volunteers can potentially execute unintentional muscle contractions (which may be also captured by $\tau_{d}(t)$ ) that can aid or hinder the desired limb motion. To mitigate this potential, volunteers were instructed to relax and to allow the stimulation to control the limb motion (i.e., the subjects were not supposed to influence the leg motion voluntarily and were not allowed to observe the desired limb trajectory).

The study volunteers were seated in a nonmotorized leg extension machine (LEM). The free swinging legs of the volunteers were attached to the movable arm of the LEM, where the position of the LEM arm is measured by an optical encoder and used as a feedback signal. Adjustments were made before each trial to ensure that the centers of the knee and the encoder were aligned. A 4.5-kg (10 lb) weight was attached on the weight bar of the LEM arm, and a mechanical stop was used to prevent hyperextension.

Self-adhesive reusable neuromuscular stimulation electrodes were used in the experiments. One electrode was placed over the distal-medial portion of the quadriceps femoris muscle groups, and the other was placed over the proximal-lateral portion. Electrical pulses were delivered through a custom-built stimulator. Data acquisition was performed at $1000 \mathrm{~Hz}$, and two digital-to-analog signals were used as inputs to the stimulation circuitry that produces a positive square pulse between 3 and $100 \mathrm{~Hz}$ with a voltage output between $1-$ and $50-\mathrm{V}$ peak.

The modulated pulsewidth was set to a constant $400 \mu \mathrm{s}$, and the frequency of the pulse sequence was $28 \mathrm{~Hz}$. The motivation for choosing a 400- $\mu$ s pulse is due to the fact that it generates reliable output based on its force-frequency and force-amplitude relationships relative to other pulsewidths. The stimulation frequency was selected based on force-frequency curves [41], which show that, as stimulation frequency is increased, muscle force increases to a saturation limit. Higher frequencies can be chosen to generate more force up to a saturation limit, but muscles tend to fatigue faster at higher frequencies. The $28-\mathrm{Hz}$ pulse wave yields reduced fatigue in comparison with higher frequencies, but lower frequencies tend to produce pulsed motion in the shank [41], [42].

\section{A. Tracking Experiments}

Tracking experiments were conducted on five volunteers (two females and three males, ages $22-40$ years). The desired angular trajectory for the knee joint was

$$
q_{d}= \begin{cases}\frac{35}{2}\left(1+\sin \left(\frac{2 \pi}{T} t+\frac{3}{2} \pi\right)\right), & t<\frac{T}{2} \\ 15\left(1+\sin \left(\frac{2 \pi}{T} t+\frac{3}{2} \pi\right)\right)+5, & t \geq \frac{T}{2}\end{cases}
$$

with a frequency of $1.5 \mathrm{~Hz}$ and range of motion between $5^{\circ}$ and $35^{\circ}$. Any sufficiently smooth desired trajectory could have been selected. For the tracking experiments, $Q$ and $R$ are adjusted by trial and error for each individual to yield the best performance. Each individual was stimulated for five to ten trials with a minimum rest of 5 min between trials. Each trial was $30 \mathrm{~s}$. The steady-state RMS and peak (i.e., $\max |e|)$ tracking errors are calculated from 3 to $30 \mathrm{~s}$. Table I summarizes the RMS and peak errors for given $Q$ and $R$ gains. Figs. 2 and 3 illustrate a typical limb tracking performance. The $Q$ and $R$ gains were adjusted to obtain the results in Table I. The mean steadystate RMS is $1.92^{\circ}$ with a standard deviation (STD) of $0.20^{\circ}$, and the mean steady-state peak error is $6.57^{\circ}$ with an STD of $1.41^{\circ}$.

Unit step tests were also conducted on three volunteers (one female and two males, ages $25-40$ years). Each individual was stimulated for five trials for $20^{\circ}$ and $40^{\circ}$, respectively. Each session was $20 \mathrm{~s}$ with a minimum rest of $5 \mathrm{~min}$ between trials. The results are summarized in Table II. A representative trial is shown in Fig. 4.

\section{B. Performance Tradeoffs}

To demonstrate the ability for a clinician to choose different combinations of $Q$ and $R$ to place a greater emphasis on tracking performance or feedback control input, tracking experiments were conducted on three healthy normal volunteers (ages 25-40 years). Two groups of experiments were conducted with fixed NN update gains. The first experiments fixed $Q=1$ and varied $R$ from 8 to 10000 to illustrate the effect of penalizing the control input. Additional experiments varied $Q$ between 8 and 600 for a fixed $R=2000$ to show the effect of penalizing 
TABLE I

RMS and PEAK ERrors For Five Healthy Normal Individuals

\begin{tabular}{|l|l|l|l|l|l|l|l|}
\hline Subject & Leg & Peak $(0-30 s)$ & RMS $(0-30 s)$ & Peak $(3-30 s)$ & RMS $(3-30 s)$ & Q & R \\
\hline A & Right & 9.67 & 2.34 & $8.91^{\circ}$ & $2.10^{\circ}$ & 44.9 & 2000 \\
\hline B & Right & 6.14 & 1.89 & $5.06^{\circ}$ & $1.81^{\circ}$ & 79.8 & 2000 \\
\hline B & Left & 9.69 & 2.20 & $6.07^{\circ}$ & $1.59^{\circ}$ & 19.9 & 2000 \\
\hline C & Left & 15.47 & 3.47 & $7.18^{\circ}$ & $2.13^{\circ}$ & 9.73 & 2000 \\
\hline D & Left & 13.72 & 3.07 & $6.88^{\circ}$ & $1.98^{\circ}$ & 19.9 & 2000 \\
\hline E & Right & 8.47 & 2.31 & $5.34^{\circ}$ & $1.91^{\circ}$ & 499.5 & 2000 \\
\hline Mean & & 10.53 & 2.54 & $6.57^{\circ}$ & $1.92^{\circ}$ & & \\
\hline STD & & 3.45 & 0.59 & $1.41^{\circ}$ & $0.20^{\circ}$ & & \\
\hline
\end{tabular}

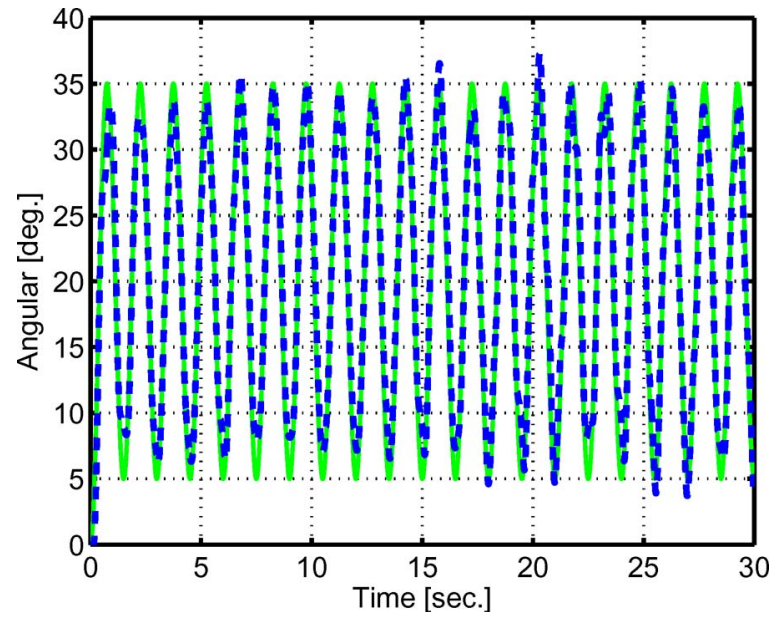

Fig. 2. Tracking performance for a representative trial. (Solid line) Desired trajectory. (Dotted line) Actual trajectory.

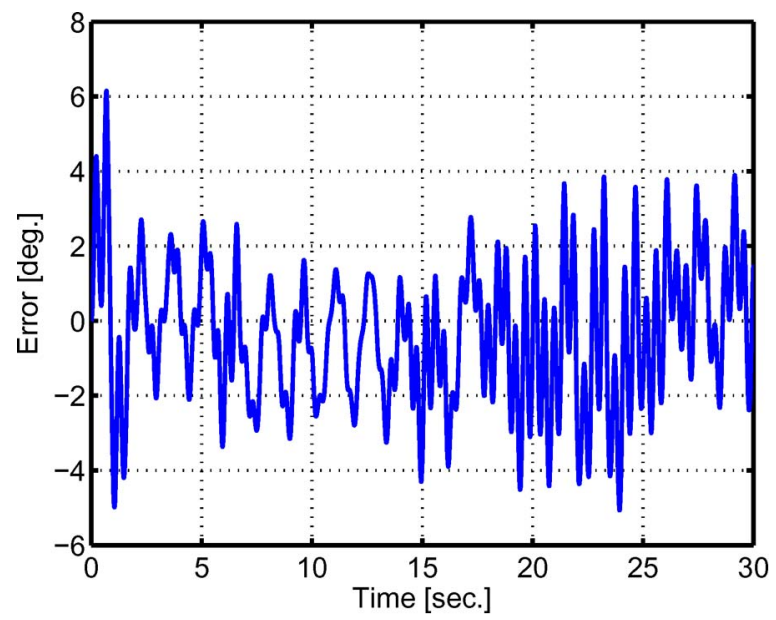

Fig. 3. Tracking error for a representative trial.

TABLE II

Step Tests For Three Healthy Normal Individuals. The Settling Time Was Set to be $10 \%$ of the Final Value. The Steady-State RMS Was Calculated From Settling Time to $20 \mathrm{~s}$

\begin{tabular}{|l|l|l|l|l|l|}
\hline Subject & Leg & Final Value & Rising Time & Settling Time & RMS \\
\hline A & Left & $20^{\circ}$ & $3.25^{\circ}$ & $3.46^{\circ}$ & $0.40^{\circ}$ \\
\hline B & Left & $20^{\circ}$ & $0.31^{\circ}$ & $0.46^{\circ}$ & $0.54^{\circ}$ \\
\hline C & Left & $20^{\circ}$ & $3.66^{\circ}$ & $3.91^{\circ}$ & $0.49^{\circ}$ \\
\hline Mean & & & $2.41^{\circ}$ & $2.61^{\circ}$ & $0.48^{\circ}$ \\
\hline A & Left & $40^{\circ}$ & $2.55^{\circ}$ & $2.80^{\circ}$ & $1.31^{\circ}$ \\
\hline B & Left & $40^{\circ}$ & $2.31^{\circ}$ & $2.54^{\circ}$ & $1.10^{\circ}$ \\
\hline C & Left & $40^{\circ}$ & $1.43^{\circ}$ & $1.62^{\circ}$ & $1.72^{\circ}$ \\
\hline Mean & & & $2.10^{\circ}$ & $2.32^{\circ}$ & $1.38^{\circ}$ \\
\hline
\end{tabular}

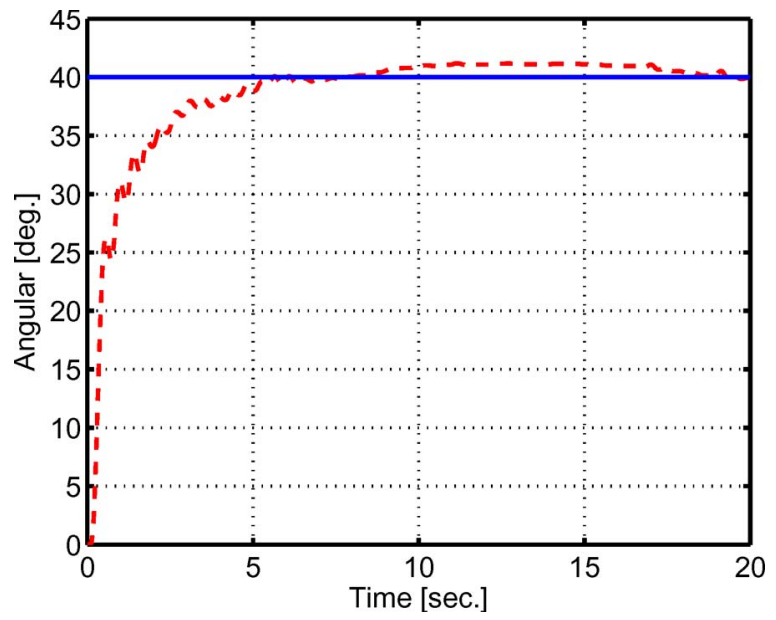

Fig. 4. Step test for a representative trial. (Solid line) Desired angle. (Dotted line) Actual trajectory.

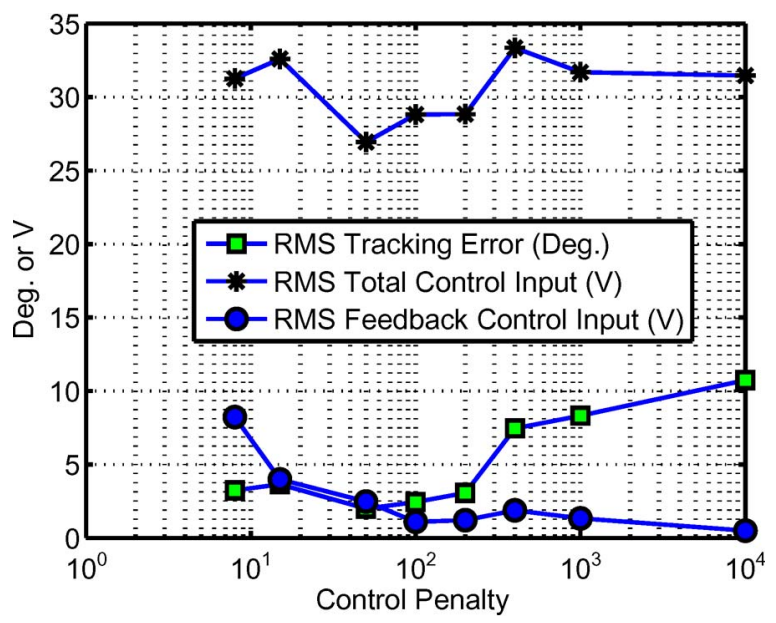

Fig. 5. Experiments with $Q=1$, where $R$ varied from 8 to 10000 .

the performance. Each session was $20 \mathrm{~s}$ with a minimum rest of 5 min between trials; and RMS values were calculated for the error, total control input, and optimal control input (i.e., $u_{1}(t)$ ), respectively. Fig. 5 illustrates that the feedback control input decreases and the tracking error increases by increasing $R$. Fig. 6 illustrates that the error decreases and the feedback control input increases with increasing $Q$. The results in Figs. 5 and 6 represent the outcome for one volunteer, but the results showed the same trends for the other two subjects with different adjustable ranges. 


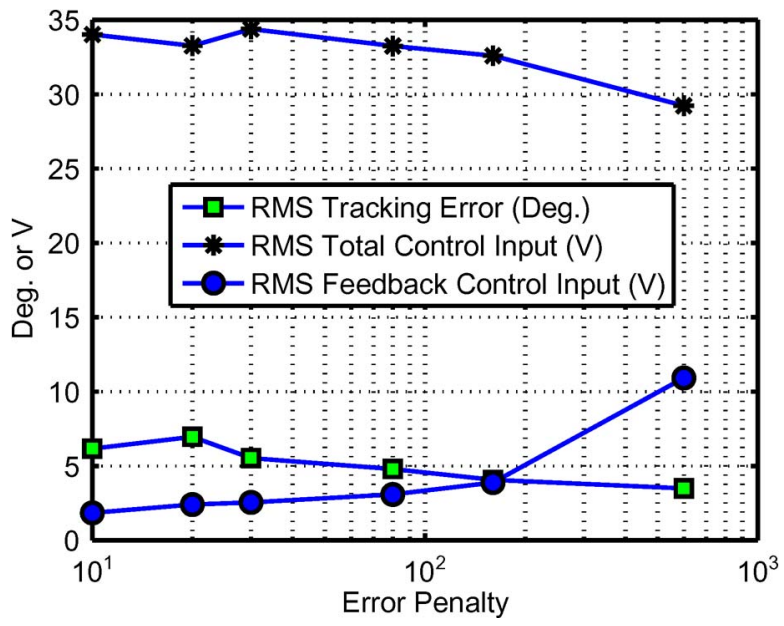

Fig. 6. Experiments where $Q$ varied from 10 to 600 and $R=2000$.

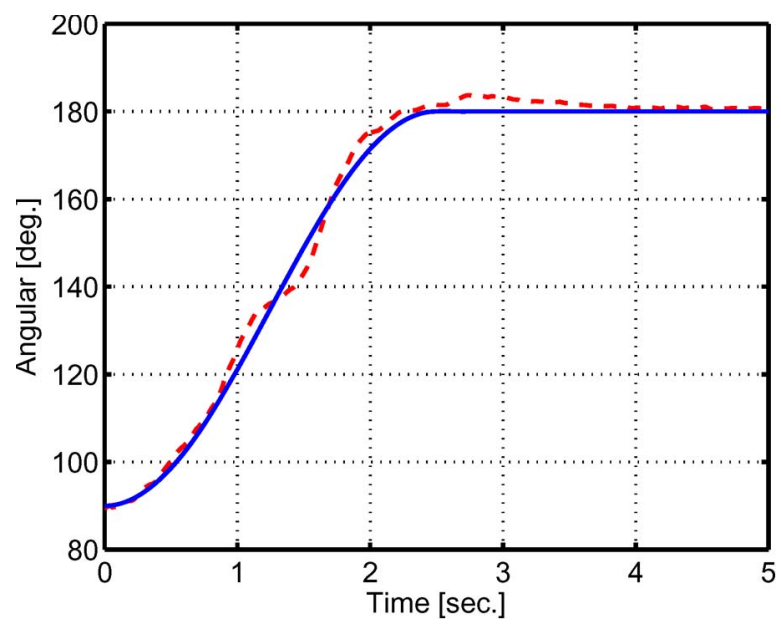

Fig. 7. Trajectories of the standing experiment. (Solid line) Desired trajectory. (Dashed line) Actual trajectory.

\section{Changing Gravity Load}

To illustrate that the proposed inverse optimal controller can be used for a task, which involves a changing load (i.e., the moment arm of gravity force changes), a sit-to-stand transitionlike experiment was conducted on a 38-year-old healthy normal male. Note that a physiological sit-to-stand transaction involves a mixed eccentric-concentric contraction of the muscles due to the biarticular nature of the quadriceps group. For this experiment, the electrodes were placed on one leg, and the volunteer was seated on the edge of a chair. The knee joint angle was measured by a goniometer (Biometrics Ltd.,VA), where the goniometer measured $90^{\circ}$ in the seated position and approximately $180^{\circ}$ in the standing position. Given the large initial condition of error (i.e., $90^{\circ}$ ), an experimentally determined desired sit-to-stand trajectory was designed as $q_{d}=$ $135^{\circ}+45^{\circ} \sin ((2 \pi / 5) t+(3 / 2) \pi)$ if $t<(5 / 2)$ and $180^{\circ}$ if $t \geq$ $(5 / 2)$. Fig. 7 depicts the actual versus desired trajectory for the standing experiment. The maximum positive and negative transient errors are $+6.0^{\circ}$ and $-5.3^{\circ}$, respectively. The steadystate error is $-0.63^{\circ} \pm 0.17^{\circ}$ with a maximum stimulation voltage of $30 \mathrm{~V}$.

\section{DISCUSSION}

An NN-based inverse optimal controller is proposed and evaluated. The controller is proven to achieve UUB tracking in the presence of bounded unmodeled disturbances. The structure of the controller is organized as a combination of an NN feedforward and a PD feedback element. The NN element compensates for the nonlinear uncertainties present in the dynamics such as passive constraints on joint movement and muscle stimulation, which include nonlinear recruitment, torque angle, torque-velocity scaling, etc. A cost functional is constructed to allow gains to be adjusted to scale the relative penalty of the tracking error or the feedback control portion of the control.

As indicated in Table I, a mean RMS error of $1.92^{\circ} \pm$ $0.2^{\circ}$ (for 3-30 s) was achieved for the given desired trajectory. The sit-to-stand transition-like experiment shows that the controller also yields promising results, where the maximum positive and negative transient errors are $+6.0^{\circ}$ and $-5.3^{\circ}$, respectively, with a steady-state error within $-0.63^{\circ} \pm 0.17^{\circ}$. The control accuracy from these experiments is sufficient for typical functional tasks. In addition to developing a controller (and associated stability proof) that can yield desired tracking error performance, a contribution of this effort is to develop a framework to adjust the performance versus control effort.

The tradeoff between tracking performance and feedback control effort can be achieved by choosing different values of $Q$ and $R$. Larger values of $Q$ yield better tracking performance at the expense of a larger feedback control effort, whereas larger values of $R$ yield reduced feedback control effort with larger tracking errors. As illustrated in Fig. 5, with $Q=1$, increasing $R$ from 8 to 10000 results in a reduction in the RMS feedback control input from 8.2 to $0.5 \mathrm{~V}$. Fig. 6 illustrates that, with $R=$ 2000 , increasing $Q$ from 20 to 600 reduces the RMS tracking error from $7^{\circ}$ to $3.5^{\circ}$. Additional development remains to examine the effects on fatigue of increased control input. Moreover, the current development is not able to include the entire control input in the cost functional (i.e., only the feedback portion of the controller is included). The results validate the ability to directly alter the feedback control through $R$, but the results do not show a correlation between changes in the feedback portion versus changes in the overall control (i.e., the overall control output was relatively invariant to changes in $R$ ). This can be explained by the $\mathrm{NN}$ feedforward component compensating for the differences. However, heuristically, it is well accepted that larger feedback gains result in noise amplification and higher frequency control. It is also well accepted that higher frequency stimulation can lead to more rapid fatigue. These results point to the need for further studies in future work to investigate the relationship between fatigue as a function of feedback control versus feedforward control. From a theoretical perspective, the approach in [43] provides an inroad to developing an inverse optimal controller that includes a portion of the feedforward component in the cost functional for the parametric strictfeedback systems.

Able-bodied individuals are a heterogeneous group with muscle size, strength, and fatigability varying greatly, which is seen in the experiment group. The results reflect robustness since the controller is able to account for individual differences 
in response to electrical stimulation. The response of muscles to electrical stimulation could be different between able-bodied individuals and individuals with various disorders. For example, a person with a spinal cord injury that occurred prior to a few weeks has muscles that are atrophied, experience more rapid fatigue, and is potentially subject to disturbances such as spasticity and clonus. The muscle atrophy and rapid fatigue can be improved through muscle reconditioning using electrical stimulation. The experimental population used in this study provides a proof of concept that the controller works to regulate electrically stimulated limb tracking, but the results should not be extrapolated to the potential performance of the system in individuals who have disorders without clinical trials in such populations.

\section{CONCLUSION}

An NN-based inverse optimal NMES controller has been developed to enable the lower limb to track a desired trajectory through electrical stimulation of the quadriceps despite uncertainties in the considered muscle activation and limb model. Experimental results for tracking a desired trajectory and a sitto-stand transition-like experiment illustrate the performance of the controller. The motivation for this result is a framework that can be used to examine the interplay between the performance and the control authority for rehabilitation clinicians. An inverse optimal method was used to ensure optimality for a derived meaningful cost functional. The framework illustrates that NN controllers augmented by a PD feedback mechanism can minimize a cost functional, which can be adjusted (e.g., through $Q$ and $R$ ) to place more emphasis on tracking error performance versus the feedback control input. While this work makes a contribution as the first analysis to explore an optimal controller for NMES, given a nonlinear uncertain muscle model, the development is limited by the restriction to use a derived cost functional. Future efforts will explore the use of implicit learning methods to solve for a value function that minimizes the cost of a given desired functional versus a derived cost, as in our preliminary work for generic nonlinear systems in [44].

\section{REFERENCES}

[1] C. Lynch and M. Popovic, "Functional electrical stimulation," IEEE Control Syst., vol. 28, no. 2, pp. 40-50, Apr. 2008.

[2] G. Lyons, T. Sinkjaer, J. Burridge, and D. Wilcox, "A review of portable FES-based neural orthoses for the correction of drop foot," IEEE Trans. Neural Syst. Rehabil. Eng., vol. 10, no. 4, pp. 260-279, Dec. 2002.

[3] L. R. Sheffler and J. Chae, "Neuromuscular electrical stimulation in neurorehabilitation," Muscle Nerve, vol. 35, no. 5, pp. 562-590, May 2007.

[4] S. Boncompagni, H. Kern, K. Rossini, C. Hofer, W. Mayr, U. Carraro, and F. Protasi, "Structural differentiation of skeletal muscle fibers in the absence of innervation in humans," Proc. Nat. Acad. Sci.USA, vol. 104, no. 49, pp. 19339-19344, Dec 2007.

[5] F. Sepulveda, M. H. Granat, and A. Cliquet, "Two artificial neural systems for generation of gait swing by means of neuromuscular electrical stimulation," Med. Eng. Phys., vol. 19, no. 1, pp. 21-28, Jan. 1997.

[6] J. J. Abbas and H. J. Chizeck, "Feedback control of coronal plane hip angle in paraplegic subjects using functional neuromuscular stimulation," IEEE Trans. Biomed. Eng., vol. 38, no. 7, pp. 687-698, Jul. 1991.

[7] N. Lan, P. E. Crago, and H. J. Chizeck, "Control of end-point forces of a multijoint limb by functional neuromuscular stimulation," IEEE Trans. Biomed. Eng., vol. 38, no. 10, pp. 953-965, Oct. 1991.
[8] N. Lan, P. E. Crago, and H. J. Chizeck, "Feedback control methods for task regulation by electrical stimulation of muscles," IEEE Trans. Biomed. Eng., vol. 38, no. 12, pp. 1213-1223, Dec. 1991.

[9] K. Stegath, N. Sharma, C. Gregory, and W. E. Dixon, "An extremum seeking method for non-isometric neuromuscular electrical stimulation," in Proc. IEEE Int. Conf. Syst. Man Cybern., Montreal, Canada, Oct. 2007, pp. 2528-2532

[10] N. Lan, H. Feng, and E. Crago, "Neural network generation of muscle stimulation patterns for control of arm movements," IEEE Trans. Rehabil. Eng., vol. 2, no. 4, pp. 213-224, Dec. 1994.

[11] J. J. Abbas and H. J. Chizeck, "Neural network control of functional neuromuscular stimulation systems: Computer simulation studies," IEEE Trans. Biomed. Eng., vol. 42, no. 11, pp. 1117-1127, Nov. 1995.

[12] D. Graupe and H. Kordylewski, "Artificial neural network control of FES in paraplegics for patient responsive ambulation," IEEE Trans. Biomed. Eng., vol. 42, no. 7, pp. 699-707, Jul. 1995.

[13] J. A. Riess and J. J. Abbas, "Adaptive neural network control of cyclic movements using functional neuromuscular stimulation," IEEE Trans. Rehabil. Eng., vol. 8, no. 1, pp. 42-52.

[14] H. Kordylewski and D. Graupe, "Control of neuromuscular stimulation for ambulation by complete paraplegics via artificial neural networks," Neurol. Res., vol. 23, no. 5, pp. 472-481, Jul. 2001.

[15] N. Sharma, C. Gregory, M. Johnson, and W. E. Dixon, "Closed-loop neural network-based NMES control for human limb tracking," IEEE Trans. Control Syst. Technol., vol. 20, no. 3, pp. 712-725, May 2012.

[16] J. P. Giuffrida and P. E. Crago, "Functional restoration of elbow extension after spinal-cord injury using a neural network-based synergistic FES controller," IEEE Trans. Neural Syst. Rehabil. Eng., vol. 13, no. 2, pp. 147152, Jun. 2005.

[17] A. Pedrocchi, S. Ferrante, E. De Momi, and G. Ferrigno, "Error mapping controller: A closed loop neuroprosthesis controlled by artificial neural networks," J. Neuroeng. Rehabil., vol. 3, no. 1, p. 25, Oct. 2006.

[18] S. Kim, M. Fairchild, A. Iarkov, J. Abbas, and R. Jung, "Adaptive control of movement for neuromuscular stimulation-assisted therapy in a rodent model," IEEE Trans. Biomed. Eng., vol. 56, no. 2, pp. 452-461, Feb. 2009.

[19] A. Ajoudani and A. Erfanian, "A neuro-sliding-mode control with adaptive modeling of uncertainty for control of movement in paralyzed limbs using functional electrical stimulation," IEEE Trans. Biomed. Eng., vol. 56, no. 7, pp. 1771-1780, Jul. 2009.

[20] J. Lujan and P. Crago, "Automated optimal coordination of multiple-DOF neuromuscular actions in feedforward neuroprostheses," IEEE Trans. Biomed. Eng., vol. 56, no. 1, pp. 179-187, Jan. 2009.

[21] N. Sharma, C. M. Gregory, M. Johnson, and W. E. Dixon, "Modified neural network-based electrical stimulation for human limb tracking," in Proc. IEEE Int. Symp. Intell. Control, San Antonio, TX, Sep. 2008, pp. $1320-1325$

[22] N. Sharma, K. Stegath, C. M. Gregory, and W. E. Dixon, "Nonlinear neuromuscular electrical stimulation tracking control of a human limb," IEEE Trans. Neural Syst. Rehabil. Eng., vol. 17, no. 6, pp. 576-584, Dec. 2009.

[23] R. Maladen, R. Perumal, A. Wexler, and S. Binder-Macleod, "Effects of activation pattern on nonisometric human skeletal muscle performance," J. Appl. Physiol., vol. 102, no. 5, pp. 1985-1991, May 2007.

[24] S. Binder-Macleod, J. Dean, and J. Ding, "Electrical stimulation factors in potentiation of human quadriceps femoris," Muscle Nerve, vol. 25, no. 2, pp. 271-279, Feb. 2002.

[25] J. Mizrahi, "Fatigue in muscles activated by functional electrical stimulation," Crit. Rev. Phys. Rehabil. Med., vol. 9, no. 2, pp. 93-129, 1997.

[26] C. M. Gregory, W. E. Dixon, and C. S. Bickel, "Impact of varying pulse frequency and duration on muscle torque production and fatigue," Muscle and Nerve, vol. 35, no. 4, pp. 504-509, Apr. 2007.

[27] R. A. Freeman and P. V. Kokotovic, Robust Nonlinear Control Design: State-Space and Lyapunov Techniques. Cambridge, MA: Birkhaülauser, 1996.

[28] M. Krstic and H. Deng, Stabilization of Nonlinear Uncertain Systems. New York: Springer-Verlag, 1998.

[29] Q. Wang, N. Sharma, M. Johnson, and W. E. Dixon, "Asymptotic optimal control of neuromuscular electrical stimulation," in Proc. IEEE Conf. Decision Control, Atlanta, GA, 2010, pp. 839-844.

[30] T. Schauer, N. O. Negard, F. Previdi, K. J. Hunt, M. H. Fraser, E. Ferchland, and J. Raisch, "Online identification and nonlinear control of the electrically stimulated quadriceps muscle," Control Eng. Pract., vol. 13, no. 9, pp. 1207-1219, Sep. 2005.

[31] M. Ferrarin and A. Pedotti, "The relationship between electrical stimulus and joint torque: A dynamic model," IEEE Trans. Rehabil. Eng., vol. 8, no. 3, pp. 342-352, Sep. 2000. 
[32] W. L. Buford, Jr., F. M. Ivey, Jr., J. D. Malone, R. M. Patterson, G. L. Peare, D. K. Nguyen, and A. A. Stewart, "Muscle balance at the knee-moment arms for the normal knee and the ACL-minus knee," IEEE Trans. Rehabil. Eng., vol. 5, no. 4, pp. 367-379, Dec. 1997.

[33] F. L. Lewis, "Neural network control of robot manipulators," IEEE Expert, vol. 11, no. 3, pp. 64-75, Jun. 1996.

[34] F. L. Lewis, R. Selmic, and J. Campos, Neuro-Fuzzy Control of Industrial Systems With Actuator Nonlinearities. Philadelphia, PA: SIAM, 2002.

[35] R. A. Freeman and P. V. Kokotovic, "Optimality of robust nonlinear feedback control," Univ. California, Santa Barbara, CA, Tech. Rep. CCEC11-09-93, Nov. 1993.

[36] M. Krstic and P. Tsiotras, "Inverse optimal stabilization of a rigid spacecraft," IEEE Trans. Autom. Control, vol. 44, no. 5, pp. 1042-1049, May 1999.

[37] W. J. Crochetiere, L. Vodovnik, and J. B. Reswick, "Electrical stimulation of skeletal muscle-A study of muscle as an actuator," Med. Biol. Eng., vol. 5, no. 2, pp. 111-125, Mar. 1967.

[38] G.-C. Chang, J.-J. Lub, G.-D. Liao, J.-S. Lai, C.-K. Cheng, B.-L. Kuo, and T.-S. Kuo, "A neuro-control system for the knee joint position control with quadriceps stimulation," IEEE Trans. Rehabil. Eng., vol. 5, no. 1, pp. 2-11, Mar. 1997.

[39] J. Hausdorff and W. Durfee, "Open-loop position control of the knee joint using electrical stimulation of the quadriceps and hamstrings," Med. Biol. Eng. Comput., vol. 29, no. 3, pp. 269-280, May 1991.

[40] K. Kurosawa, R. Futami, T. Watanabe, and N. Hoshimiya, "Joint angle control by FES using a feedback error learning controller," IEEE Trans. Neural Syst. Rehabil. Eng., vol. 13, no. 3, pp. 359-371, Sep. 2005.

[41] R. Riener and J. Quintern, Biomechanics and Neural Control of Posture and Movement, J. Winters and P. E. Crago, Eds. New York: SpringerVerlag, 2000.

[42] R. Riener, J. Quintern, and G. Schmidt, "Biomechanical model of the human knee evaluated by neuromuscular stimulation," J. Biomech., vol. 29, no. 9, pp. 1157-1167, Sep. 1996.

[43] Z. H. Li and M. Krstic, "Optimal design of adaptive tracking controllers for non-linear systems," Automatica, vol. 33, no. 8, pp. 1459-1473, Aug. 1997.

[44] K. Dupree, P. Patre, Z. Wilcox, and W. E. Dixon, "Asymptotic optimal control of uncertain nonlinear systems," Automatica, vol. 47, no. 1, pp. 99-107, Jan. 2011

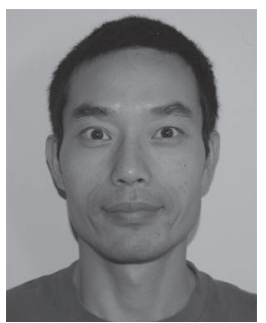

Qiang Wang received the Ph.D. degree in biomedical engineering from Peking Union Medical College and Chinese Academy of Medical Sciences, Beijing, China, in 2003, for the biomedical applications of near-infrared spectroscopy. He is currently working toward the Ph.D. degree in electrical and computer engineering at the University of Florida, Gainesville.

He was a Postdoctoral Associate with the Department of Biomedical Engineering, University of Florida, in 2005-2008, doing research in biomedical optics. His main research interests are Lyapunovbased nonlinear control theory and the applications for complex biomedical systems.

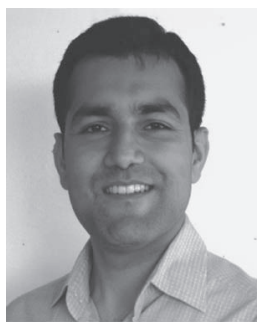

Nitin Sharma received the Ph.D. degree from the University of Florida, Gainesville, in 2010.

After his doctoral studies, he was an Alberta Innovates-Health Solutions Postdoctoral Fellow with the Department of Physiology, University of Alberta, Edmonton, Canada. In 2012, he joined the Department of Mechanical Engineering and Material Science, University of Pittsburgh, Pittsburgh, PA, as an Assistant Professor. His research interests include intelligent and robust control of functional electrical stimulation (FES); modeling, optimization, and control of FES-elicited walking; and control of uncertain nonlinear systems with input and state delays.

Dr. Sharma was a recipient of the O. Hugo Schuck Award in 2009 and the Best Student Paper Award in Robotics at the 2009 American Society of Mechanical Engineers Dynamic Systems and Controls Conference. He was also a finalist for the Best Student Paper Award at the 2008 IEEE Multiconference on Systems and Control.

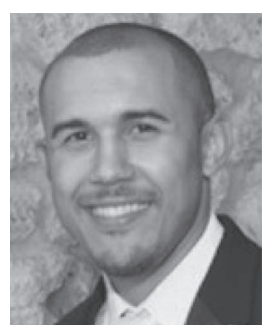

Marcus Johnson (M'09) received the Ph.D. degree from the University of Florida, Gainesville, in 2011.

$\mathrm{He}$ is currently a Research Aerospace Engineer with the Ames Research Center, National Aeronautics and Space Administration, Moffett Field, CA. His main research interest is the development of Lyapunov-based proofs for optimality of nonlinear adaptive systems.

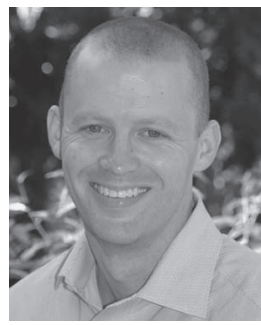

Chris M. Gregory received his Ph.D. degree in Exercise Physiology from the University of Georgia.

$\mathrm{He}$ is a Research Health Scientist with the Ralph H. Johnson VAMC, Charleston, SC, and a Faculty Member with the Department of Health Sciences and Research, The Medical University of South Carolina, Charleston. His main research interest has been the development interventions to treat neuromuscular dysfunction in persons with central nervous system injury or disease. The primary goal of this line of research is the identification of mechanisms (physiological and behavioral) that contribute to (or limit) functional improvements. His research has been funded by the Rehabilitation Research and Development Service of the Veterans Health Administration, the National Institutes of Health, the American Heart Association, and the South Carolina Spinal Cord Injury Research Fund.

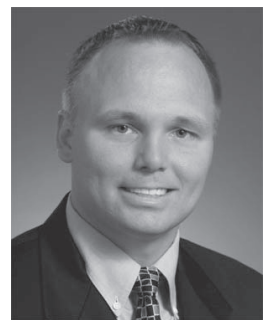

Warren E. Dixon (M'94-SM'05) received his Ph.D degree in 2000 from the Department of Electrical and Computer Engineering from Clemson University.

In 2004, he joined the Mechanical and Aerospace Engineering Department Faculty, University of Florida, Gainesville, where he is currently a Full Professor and a Charles Taylor Faculty Fellow (2012-2015) and where he holds a University of Florida Research Foundation Professorship (2012-2015). He has authored or coauthored three books, an edited collection, 9 chapters, and over 250 refereed journal and conference papers. His main research interest has been the development and application of Lyapunov-based control techniques for uncertain nonlinear systems

Dr. Dixon served as an Appointed Member of the IEEE Control Systems Society Board of Governors (BoG) in 2008 and now serves as the Director of Operations for the Executive Committee of the BoG. He is currently or formerly an Associate Editor of the American Society of Mechanical Engineers (ASME)Automatica; the IEEE Transactions on Systems Man and Cybernetics-Part B: Cybernetics; and the International Journal of Robust and Nonlinear Control. His work has been recognized by the 2011 ASME Dynamics Systems and Control Division Outstanding Young Investigator Award, the 2009 American Automatic Control Council O. Hugo Schuck Award, the 2006 IEEE Robotics and Automation Society Early Academic Career Award, the National Science Foundation CAREER Award (2006-2011), the 2004 U.S Department of Energy Outstanding Mentor Award, and the 2001 Oak Ridge National Laboratory Early Career Award for Engineering Achievement. 